\title{
Heart Rate Variability in Postoperative Patients with Nonfunctioning Pituitary Adenoma
}

Jeonghoon Ha ${ }^{1}$, Hansang Baek ${ }^{1}$, Chaiho Jeong ${ }^{1}$, Minsoo Yeo², Seung-Hwan Lee ${ }^{1}$, Jae Hyoung Cho ${ }^{1}$, Ki-Hyun Baek ${ }^{3}$, Moo Il Kang ${ }^{1}$, Dong-Jun Lim ${ }^{1}$

${ }^{1}$ Division of Endocrinology and Metabolism, Department of Internal Medicine, Seoul St. Mary's Hospital, College of Medicine, The Catholic University of Korea, Seoul; ${ }^{2}$ Digital Healthcare Research Center, Taewoong Medical Co. Ltd., Gimpo; ${ }^{3}$ Division of Endocrinology and Metabolism, Department of Internal Medicine, Yeouido St. Mary's Hospital, College of Medicine, The Catholic University of Korea, Seoul, Korea

Background: Decreased heart rate variability (HRV) has been reported to be associated with cardiac autonomic dysfunction. Hypopituitarism in nonfunctioning pituitary adenoma (NFPA) is often linked to increased cardiovascular mortality. We therefore hypothesized that postoperative NFPA patients with hormone deficiency have an elevated risk of HRV alterations indicating cardiac autonomic dysfunction.

Methods: A total of 22 patients with NFPA were enrolled in the study. Between 3 and 6 months after surgery, a combined pituitary function test (CPFT) was performed, and HRV was measured. The period of sleep before the CPFT was deemed the most stable period, and the hypoglycemic period that occurred during the CPFT was defined as the most unstable period. Changes in HRV parameters in stable and unstable periods were observed and compared depending on the status of hormone deficiencies.

Results: In patients with adrenocorticotropic hormone (ACTH) deficiency with other pituitary hormone deficiencies, the low frequency to high frequency ratio, which represents overall autonomic function and is increased in the disease state, was higher $(P=0.005)$. Additionally, the standard deviation of the normal-to-normal interval, which decreases in the autonomic dysfunction state, was lower $(P=0.030)$ during the hypoglycemic period. In panhypopituitarism, the low frequency to high frequency ratio during the hypoglycemic period was increased $(P=0.007)$.

Conclusion: HRV analysis during CPFT enables estimation of cardiac autonomic dysfunction in patients with NFPA who develop ACTH deficiency with other pituitary hormone deficiencies or panhypopituitarism after surgery. These patients may require a preemptive assessment of cardiovascular risk.

Keywords: Heart rate; Pituitary neoplasms; Pituitary hormones; Autonomic nervous system

\section{INTRODUCTION}

Nonfunctioning pituitary adenoma (NFPA) is defined as a benign pituitary tumor that does not result in hormone hypersecre-

Received: 28 January 2021, Revised: 8 March 2021, Accepted: 29 March 2021

Corresponding author: Dong-Jun Lim

Division of Endocrinology and Metabolism, Department of Internal Medicine, Seoul St. Mary's Hospital, College of Medicine, The Catholic University of Korea, 222 Banpo-daero, Seocho-gu, Seoul 06591, Korea

Tel: +82-2-2258-6009, Fax: +82-2-599-3589, E-mail: 1dj6026@catholic.ac.kr tion; this type of neoplasm accounts for one-third of all tumors in the pituitary gland [1]. NFPA is associated with the development of hypopituitarism because of the mass effect of the growing tumor or alongside postoperative irradiation, which increas-

Copyright $\odot 2021$ Korean Endocrine Society

This is an Open Access article distributed under the terms of the Creative Commons Attribution Non-Commercial License (https://creativecommons.org/ licenses/by-nc/4.0/) which permits unrestricted non-commercial use, distribution, and reproduction in any medium, provided the original work is properly cited. 
es the risk of hypopituitarism [1].

The effect of pituitary adenoma on mortality has been studied mainly in functioning pituitary adenoma, such as acromegaly and Cushing's disease [2,3]. Cardiometabolic risk is increased due to hypersecretion of hormones, as is mortality $[2,4]$. However, there are conflicting results concerning hormone-related mortality changes in NFPA. Several studies have reported on the mortality of NFPA patients; however, the results vary according to the demographic distribution of the study subjects and the type of hormone deficiency [2,4-6]. O'Reilly et al. [7] reported that mortality increased as the severity of hypopituitarism increased, and adrenocorticotropic hormone (ACTH) deficiency increased the mortality risk 2.28 -fold (95\% confidence interval, 1.16 to 4.49 ) in an analysis of a cohort involving 519 cases of NFPA. ACTH deficiency has been reported to adversely affect the cardiovascular system [8], but physiological nature of this effect has not yet been determined.

Heart rate (HR) is a representative biomarker in various medical conditions [9]. Heart rate variability (HRV) is a useful health indicator; studies have been conducted on HRV changes in many disease states [10], and there is robust evidence for HRV analysis as a tool reflecting cardiac autonomic function. However, there was no report confirming the change in HRV according to the postoperative hormonal deficiency pattern in NFPA patients. Therefore, we intend to analyze the effect on cardiac autonomic function by evaluating the HRV change according to the postoperative hormonal deficiency pattern in NFPA patients.

\section{METHODS}

\section{Subjects}

This prospective study enrolled 22 patients with NFPA who were referred to the endocrinologist for postoperative follow-up during the period between January 2018 and August 2019. All patients underwent surgery using the endoscopic endonasal transsphenoidal approach (EETSA) from two neurosurgeons at our institution (Yong-Kil Hong, Sin-Soo Jeon) with more than 20 years of experience each. Patients with other underlying diseases or taking any medications for therapeutic purpose were not enrolled in the study. In addition, patients who relapsed after a previous treatment or those whose pituitary stalk was removed during the operation were not enrolled. Since the purpose of our study was to identify HRV changes according to the pattern of hormone deficiency after transsphenoidal approach (TSA) in patients with NFPA, the pituitary hormone function of patients with postoperative NFPA was evaluated using a combined pituitary function test
(CPFT) 3 to 6 months after TSA; none of the subjects had any restrictions on performing the CPFT. Patients were placed in the deficient group if their hormones did not respond normally in the CPFT, and the preserved group indicated patients with a normal response as observed by the CPFT. The groups varied according to the type of hormone. Informed consent was obtained from all study participants, and the study was approved by the Institutional Review Board of Seoul St. Mary's Hospital (KC17OESI0205) and registered with Clinicaltrials.org (NCT03164148).

\section{Heart rate variability}

HRV data were recorded using an electrocardiographic sensor with a disposable patch-type electrode (T-Rex TR100A, TaeWoong Medical, Gimpo, Korea). The device has previously been used in other HRV-related studies, and the stability of data collection with this apparatus has been verified [11-13]. The device was attached to the participant's anterior chest (Supplemental Fig. S1), and there were no restrictions on daily movement, although a supine position was maintained during sleep. All patients were requested to maintain a supine position during the CPFT. Collected row data were transformed into HRV data via Kubios HRV software [14], and the results were corrected for missing or incorrect peaks. HRV parameters were generated as recommended by the Task Force of the European Society of Cardiology and the North American Society of Pacing and Electrophysiology [9]. Parameter adaptation was performed in the same manner recently described by Park et al. [13]. For timedomain analysis, the mean HR and the standard deviation of the normal-to-normal interval (SDNN) were measured. For the frequency domain, low frequency (LF) $(0.04$ to $0.15 \mathrm{~Hz})$, high frequency (HF) (0.15 to $0.40 \mathrm{~Hz}$ ), and the LF/HF ratio were analyzed. HF is an indicator reflecting parasympathetic effects, while LF is used as a reflection of sympathetic activity [10]. The LF/HF ratio and SDNN represent the subject's overall autonomic function [10]. HF decreases in the disease state [15]. In the event of autonomic dysfunction, the LF/HF ratio increases and SDNN decreases [15].

\section{Combined pituitary function test}

Changes in hormonal function were evaluated via the CPFT, which was performed between three and six months after the surgery. The CPFT was performed after an overnight fast, and the results of testing were confirmed by two endocrinologists (C.J., J.H.). After intravenous injection of $0.1 \mathrm{U} / \mathrm{kg}$ regular insulin, $200 \mu \mathrm{g}$ thyrotropin-releasing hormone, and $100 \mu \mathrm{g}$ luteinizing hormone (LH)-releasing hormone, the responses of serum 
growth hormone $(\mathrm{GH})$, cortisol, ACTH, prolactin (PRL), thyroid-stimulating hormone (TSH), $\mathrm{LH}$, and follicle-stimulating hormone (FSH) were collected serially for 2 hours (at 0, 30, 60, 90, and 120 minutes). Hypoglycemia that occurred after administration of regular insulin was deemed valid when serum glucose measured after 30 minutes of intravenous insulin was reduced to $40 \mathrm{mg} / \mathrm{dL}$ or less or by $50 \%$ or more compared to baseline. The test results were interpreted as previously described $[16,17]$. The criteria for normal responses were as follows: the peak GH level increased above $3 \mathrm{ng} / \mathrm{mL}$; the peak cortisol level increased above $18 \mu \mathrm{g} / \mathrm{dL}$ or increased by more than $7 \mu \mathrm{g} / \mathrm{dL}$ compared to the basal level; the peak TSH level increased by more than $5 \mu \mathrm{IU} / \mathrm{mL}$ compared to the basal level, while free thyroxine was within the normal range; the basal PRL level ranged from 2 to $15 \mathrm{ng} / \mathrm{mL}$, and the peak PRL level was more than twice the basal PRL level. For gonadotropin (Gn), an increase in FSH by more than $2 \mathrm{mIU} / \mathrm{mL}$ and an increase in LH by more than $10 \mathrm{mIU} / \mathrm{mL}$ compared with the basal level were considered normal responses. When a particular hormone did not show a normal response, it was deemed to be deficient, and when the normal response was confirmed, the hormone was deemed to be preserved. The presence of multiple hormone deficiencies was defined as deficiencies of two or three individual anterior pituitary hormonal axes. Panhypopituitarism was defined as the coexistence of GH, ACTH, TSH, and $\mathrm{Gn}$ deficiencies. None of the patients were receiving growth or sex hormones prior to CPFT. If their cortisol level measured at 8:00 AM after TSA surgery was less than $3 \mu \mathrm{g} / \mathrm{dL}$ or if they exhibited symptoms related to cortisol deficiency, hydrocortisone was administered. However, hydrocortisone was discontinued at least 24 hours before CPFT was performed.

\section{Study flow}

The flow of the study is depicted in Fig. 1. The CPFT examination was complex, and the procedure was delicate; therefore, only one subject was tested at a time, and a designated nurse with more than 5 years of experience in the endocrinology ward was in charge of the testing. For the CPFT, the subject was admitted to the ward by 5:00 PM the day before the examination. At 6:00 PM, the HRV device was mounted on the patient's anterior chest, and the patient was allowed to ambulate without any limitations. At 10:00 PM, the subject was asked to go to sleep and to fast until the next day's examination. The HRV device continuously recorded the patient's HRV, and the data were observed closely at 3:00 AM (baseline), when the patient was sleeping and was considered to be in the most stable state. Stay-

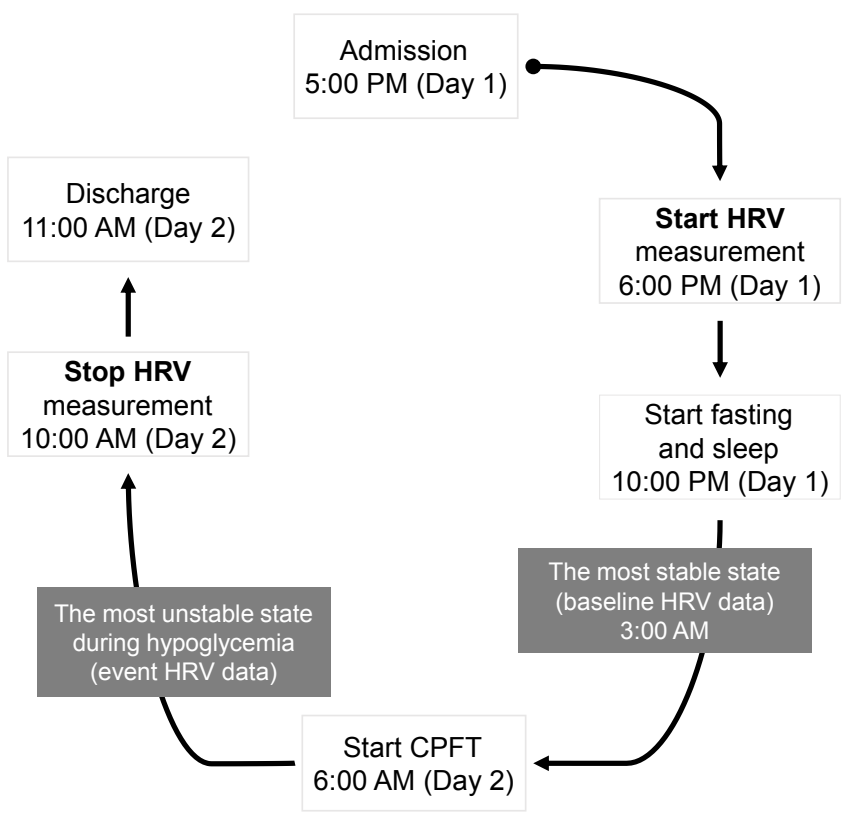

Fig. 1. Study flow of the enrolled subjects. HRV, heart rate variability; CPFT, combined pituitary function test.

ing at the hospital is an unusual experience for patients; hence, it may not be psychologically comfortable. This may be different when compared to a situation where the patient can stay comfortably at home. However, it is difficult to control the environment they would be exposed to at home; therefore, even if hospitalization causes anxiety, it was important for the patients to be in an environmentally controlled setting for the study. Preparation for the CPFT began at 6:00 AM on the day of examination, and hypoglycemia was induced between 6:30 AM and 7:00 AM according to the protocol. The period when hypoglycemia occurred was considered the most unstable state, and HRV data during this period were intensively observed. After the examination, the subjects were discharged at 10:00 AM, and HRV recording was terminated. After the HRV device was removed, all data collected from the patient were transferred to Kubios HRV software and analyzed.

\section{Statistical analysis}

Continuous data are expressed as either the mean \pm standard deviation or the median (interquartile range $[\mathrm{IQR}]$ ). Categorical data are expressed as frequencies (proportions, \%). A chi-square test or Fisher's exact test was used for categorical data as appropriate. Continuous variables were compared between the two groups by Student's $t$ test or Mann-Whitney $U$ test. Continuous data were evaluated for statistically significant differences using 
Table 1. Baseline Characteristics of the Study Subjects $(n=22)$

\begin{tabular}{|c|c|}
\hline Characteristic & Value \\
\hline Age, yr & $53.8 \pm 16.6$ \\
\hline Male sex & $10(45.5)$ \\
\hline Fasting glucose, mg/dL & $94.3 \pm 7.5$ \\
\hline Serum glucose at nadir, $\mathrm{mg} / \mathrm{dL}$ & $37.7 \pm 4.0$ \\
\hline SBP, mm Hg & $120.5 \pm 11.8$ \\
\hline DBP, $\mathrm{mm} \mathrm{Hg}$ & $71.3 \pm 8.6$ \\
\hline Time since surgery, mo & $4.4(3.2-5.9)$ \\
\hline Maximal tumor size, $\mathrm{mm}$ & $25.9(13-43)$ \\
\hline Pathology of pituitary tumor & Adenoma (100) \\
\hline \multicolumn{2}{|l|}{ Hormone deficiency after surgery ${ }^{a}$} \\
\hline $\mathrm{GH}$ & $11(50.0)$ \\
\hline ACTH & $12(54.5)$ \\
\hline TSH & $9(40.9)$ \\
\hline Prolactin & $6(27.2)$ \\
\hline FSH & $13(59.1)$ \\
\hline LH & $12(54.5)$ \\
\hline \multicolumn{2}{|l|}{ Multiple hormone deficiency } \\
\hline Single deficiency & 0 \\
\hline Multiple deficiency & $10(45.5)$ \\
\hline Panhypopituitarism & $12(54.5)$ \\
\hline \multicolumn{2}{|l|}{ Baseline HRV parameter } \\
\hline Heart rate at resting & $59.7 \pm 2.0$ \\
\hline $\mathrm{LF}, \mathrm{ms}^{2}$ & $591.9 \pm 127.9$ \\
\hline $\mathrm{HF}, \mathrm{ms}^{2}$ & $276.4 \pm 57.6$ \\
\hline $\mathrm{LF} / \mathrm{HF}$ ratio & 2.9 \\
\hline SDNN, ms & $58.3 \pm 5.2$ \\
\hline
\end{tabular}

Values are expressed as mean \pm standard deviation, number (\%), or median (interquartile range).

SBP, systolic blood pressure; DBP, diastolic blood pressure; GH, growth hormone; ACTH, adrenocorticotropic hormone; TSH, thyroid-stimulating hormone; FSH, follicle-stimulating hormone; LH, luteinizing hormone; HRV, heart rate variability; LF, low frequency; HF, high frequency; SDNN, standard deviation of normal-to-normal interval.

${ }^{\mathrm{a} C}$ Confirmed by combined pituitary function test performed at median

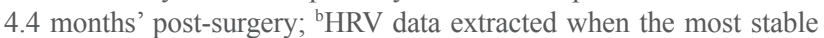
state of sleep at 3:00 AM.

a paired $t$ test or Wilcoxon signed-rank test as appropriate. Either a repeated-measures analysis of variance or a Friedman test was used to explore whether there were significant changes in HRV parameters, and a value of $P<0.05$ was considered statistically significant. Statistical analyses were performed using IBM SPSS Statistics software for Windows version 24.0 (IBM Corp., Armonk, NY, USA) and R software version 3.4.3 (R Founda-
Table 2. Differences in Parameters of Heart Rate Variability during Sleep (Most Stable State) Depending on the Stimulation State of Pituitary Hormones

\begin{tabular}{|c|c|c|c|}
\hline Parameter & Deficient & Preserved & $P$ value \\
\hline GH & 11 & 11 & \\
\hline Heart rate & $58.2 \pm 2.8$ & $61.7 \pm 3.0$ & 0.403 \\
\hline $\mathrm{LF}$ & $403.0 \pm 100.8$ & $794.9 \pm 252.8$ & 0.222 \\
\hline $\mathrm{HF}$ & $203.8 \pm 60.5$ & $369.7 \pm 100.7$ & 0.188 \\
\hline LF/HF ratio & 3.2 & 2.4 & 0.349 \\
\hline SDNN & $52.5 \pm 5.1$ & $65.7 \pm 9.6$ & 0.254 \\
\hline ACTH & 12 & 10 & \\
\hline Heart rate & $58.6 \pm 2.2$ & $61.0 \pm 3.7$ & 0.568 \\
\hline $\mathrm{LF}$ & $558.4 \pm 142.8$ & $635.0 \pm 240.9$ & 0.790 \\
\hline $\mathrm{HF}$ & $210.1 \pm 176.1$ & $361.6 \pm 104.5$ & 0.236 \\
\hline LF/HF ratio & 3.4 & 2.1 & 0.076 \\
\hline SDNN & $56.1 \pm 5.8$ & $61.1 \pm 9.6$ & 0.663 \\
\hline TSH & 9 & 13 & \\
\hline Heart rate & $55.9 \pm 3.1$ & $61.4 \pm 2.5$ & 0.218 \\
\hline $\mathrm{LF}$ & $651.9 \pm 244.2$ & $564.7 \pm 156.9$ & 0.772 \\
\hline $\mathrm{HF}$ & $200.8 \pm 84.7$ & $310.7 \pm 74.6$ & 0.352 \\
\hline LF/HF ratio & 3.9 & 2.4 & 0.145 \\
\hline SDNN & $59.9 \pm 9.0$ & $57.5 \pm 6.6$ & 0.837 \\
\hline Prolactin & 6 & 16 & \\
\hline Heart rate & $58.8 \pm 4.7$ & $60.1 \pm 7.2$ & 0.786 \\
\hline LF & $656.1 \pm 242.0$ & $562.8 \pm 157.5$ & 0.748 \\
\hline $\mathrm{HF}$ & $197.8 \pm 85.9$ & $312.1 \pm 74.1$ & 0.376 \\
\hline LF/HF ratio & 4.2 & 2.2 & 0.143 \\
\hline SDNN & $58.6 \pm 9.8$ & $58.1 \pm 6.4$ & 0.971 \\
\hline FSH & 13 & 9 & \\
\hline Heart rate & $59.6 \pm 2.6$ & $59.8 \pm 3.4$ & 0.976 \\
\hline $\mathrm{LF}$ & $591.2 \pm 176.8$ & $593.1 \pm 192.8$ & 0.994 \\
\hline $\mathrm{HF}$ & $231.1 \pm 74.9$ & $351.9 \pm 88.9$ & 0.320 \\
\hline LF/HF ratio & 3.3 & 2.1 & 0.127 \\
\hline SDNN & $56.8 \pm 7.0$ & $60.7 \pm 8.1$ & 0.726 \\
\hline LH & 12 & 10 & \\
\hline Heart rate & $59.1 \pm 2.8$ & $60.4 \pm 3.0$ & \\
\hline $\mathrm{LF}$ & $633.3 \pm 192.0$ & $538.7 \pm 171.8$ & 0.719 \\
\hline $\mathrm{HF}$ & $245.2 \pm 82.3$ & $316.4 \pm 83.1$ & 0.553 \\
\hline $\mathrm{LF} / \mathrm{HF}$ ratio & 3.4 & 2.1 & 0.095 \\
\hline SDNN & $58.7 \pm 7.5$ & $57.7 \pm 7.4$ & 0.929 \\
\hline
\end{tabular}

Values are expressed as mean \pm standard error. Hormone stimulations were confirmed by combined pituitary function test performed at median 4.4 months' post-surgery. Unit for LF and HF, $\mathrm{ms}^{2}$ and for SDDN, ms. $P<0.05$ between the two groups.

$\mathrm{GH}$, growth hormone; LF, low frequency; HF, high frequency; SDNN, standard deviation of normal-to-normal interval; ACTH, adrenocorticotropic hormone; TSH, thyroid-stimulating hormone; FSH, follicle-stimulating hormone; $\mathrm{LH}$, luteinizing hormone. 
tion for Statistical Computing, Vienna, Austria).

\section{RESULTS}

\section{Baseline characteristics}

Clinical characteristics at baseline are summarized in Table 1. The mean age of the subjects was $53.8 \pm 16.6$ years. The serum glucose level after 8 hours of overnight fasting was $94.3 \pm 7.5$ $\mathrm{mg} / \mathrm{dL}$, and the nadir of serum glucose during the CPFT was $37.7 \pm 4.0 \mathrm{mg} / \mathrm{dL}$. The interval between surgery and the CPFT was 4.4 months (IQR, 3.2 to 5.9). The longest axis of the pituitary mass measured $25.9 \mathrm{~mm}$ (IQR, 13 to 43 ). The most commonly identified deficiency was Gn deficiency (FSH deficiency $59.1 \%$ and LH deficiency 54.5\%), followed by ACTH deficiency $(54.5 \%)$ and GH deficiency (50.0\%). No single hormone deficiency was observed, and panhypopituitarism was observed in 12 patients (54.5\%). The baseline LF/HF ratio was 2.9 , and the SDNN was $58.3 \pm 5.2 \mathrm{~ms}$.

\section{HRV changes during the most stable period}

The difference in HRV parameters for each pituitary hormone deficiency during sleep (the most stable period) is presented in Table 2. Although the difference was not significant, the HR was found to be low in the deficient group regardless of the type of hormone deficiency. In the deficient group, the HF, which decreases in the disease state, was lower, and the LF/HF ratio, which represents overall autonomic function and increases in the event of autonomic dysfunction, was higher than that of the preserved group. This pattern was consistently observed regardless of the type of hormone deficiency (Table 2).

\section{HRV changes in the most unstable period}

Changes in HRV parameters for each pituitary hormone deficiency during the hypoglycemic state (the most unstable period) are summarized in Table 3. The HR was found to be low in the deficient group regardless of the type of hormone deficiency, but no significant difference was observed. HF and SDNN were lower in patients with impaired ACTH response than in those with normal ACTH response (HF: $87.5 \pm 39.9$ in the deficient group vs. $193.7 \pm 55.2$ in the preserved group, $P=0.022$; SDNN: $40.1 \pm 4.2$ in the deficient group vs. $49.8 \pm 9.6$ in the preserved group, $P=0.030$ ) (Table 3 ). The $\mathrm{LF} / \mathrm{HF}$ ratio was significantly higher in the deficient group (6.9) than in the preserved group (1.9) $(P=0.005)$ (Table 3). As shown in Fig. 2, in patients with impaired ACTH response (deficit group), HF (Fig. 2B), and SDNN (Fig. 2D) were significantly lowered, and the LF/HF ra-
Table 3. Differences in Parameters of Heart Rate Variability during hypoglycemia (Most Unstable State) Depending on the Stimulation State of Pituitary Hormones

\begin{tabular}{|c|c|c|c|}
\hline Parameter & Deficient & Preserved & $P$ value \\
\hline GH & 11 & 11 & \\
\hline Heart rate & $64.7 \pm 3.6$ & $74.4 \pm 4.6$ & 0.111 \\
\hline $\mathrm{LF}$ & $226.0 \pm 49.2$ & $323.9 \pm 119.7$ & 0.471 \\
\hline $\mathrm{HF}$ & $111.9 \pm 41.6$ & $162.2 \pm 60.2$ & 0.490 \\
\hline $\mathrm{LF} / \mathrm{HF}$ ratio & 4.9 & 4.6 & 0.884 \\
\hline SDNN & $41.2 \pm 4.2$ & $48.4 \pm 9.8$ & 0.522 \\
\hline АCTH & 12 & 10 & \\
\hline Heart rate & $68.6 \pm 4.8$ & $69.2 \pm 4.1$ & 0.927 \\
\hline $\mathrm{LF}$ & $242.1 \pm 47.9$ & $303.2 \pm 122.9$ & 0.656 \\
\hline $\mathrm{HF}$ & $87.5 \pm 39.9$ & $193.7 \pm 55.2$ & 0.022 \\
\hline $\mathrm{LF} / \mathrm{HF}$ ratio & 6.9 & 1.9 & 0.005 \\
\hline SDNN & $40.1 \pm 4.2$ & $49.8 \pm 9.6$ & 0.030 \\
\hline TSH & 9 & 13 & \\
\hline Heart rate & $65.5 \pm 6.4$ & $70.5 \pm 3.4$ & 0.469 \\
\hline $\mathrm{LF}$ & $192.5 \pm 48.2$ & $303.6 \pm 81.1$ & 0.259 \\
\hline $\mathrm{HF}$ & $77.7 \pm 55.9$ & $159.5 \pm 42.8$ & 0.288 \\
\hline $\mathrm{LF} / \mathrm{HF}$ ratio & 6.4 & 4.0 & 0.281 \\
\hline SDNN & $38.0 \pm 1.2$ & $47.25 \pm 6.9$ & 0.213 \\
\hline Prolactin & 6 & 16 & \\
\hline Heart rate & $68.5 \pm 6.9$ & $69.1 \pm 3.3$ & 0.930 \\
\hline LF & $203.3 \pm 39.1$ & $298.7 \pm 82.7$ & 0.315 \\
\hline $\mathrm{HF}$ & $84.0 \pm 54.2$ & $156.6 \pm 43.7$ & 0.348 \\
\hline $\mathrm{LF} / \mathrm{HF}$ ratio & 5.8 & 4.3 & 0.473 \\
\hline SDNN & $37.4 \pm 1.7$ & $47.6 \pm 6.8$ & 0.174 \\
\hline FSH & 13 & 9 & \\
\hline Heart rate & $68.5 \pm 4.4$ & $69.2 \pm 4.2$ & 0.908 \\
\hline $\mathrm{LF}$ & $232.9 \pm 60.0$ & $328.8 \pm 123.1$ & 0.444 \\
\hline $\mathrm{HF}$ & $98.7 \pm 39.1$ & $192.7 \pm 62.3$ & 0.198 \\
\hline LF/HF ratio & 6.0 & 2.7 & 0.111 \\
\hline SDNN & $39.8 \pm 4.7$ & $52.0 \pm 9.9$ & 0.230 \\
\hline LH & 12 & 10 & \\
\hline Heart rate & $67.9 \pm 4.5$ & $70.2 \pm 4.2$ & 0.716 \\
\hline LF & $243.0 \pm 66.2$ & $302.1 \pm 107.4$ & 0.631 \\
\hline $\mathrm{HF}$ & $107.5 \pm 42.6$ & $167.9 \pm 58.2$ & 0.406 \\
\hline $\mathrm{LF} / \mathrm{HF}$ ratio & 5.4 & 3.9 & 0.444 \\
\hline SDNN & $41.2 \pm 5.0$ & $48.5 \pm 9.1$ & 0.471 \\
\hline
\end{tabular}

Values are expressed as mean \pm standard error. Hormone stimulations were confirmed by combined pituitary function test performed at median 4.4 months' post-surgery. Unit for LF and $\mathrm{HF}, \mathrm{ms}^{2}$ and for SDDN, ms. $P<0.05$ between the two groups.

$\mathrm{GH}$, growth hormone; LF, low frequency; HF, high frequency; SDNN, standard deviation of normal-to-normal interval; ACTH, adrenocorticotropic hormone; TSH, thyroid-stimulating hormone; FSH, follicle-stimulating hormone; $\mathrm{LH}$, luteinizing hormone. 

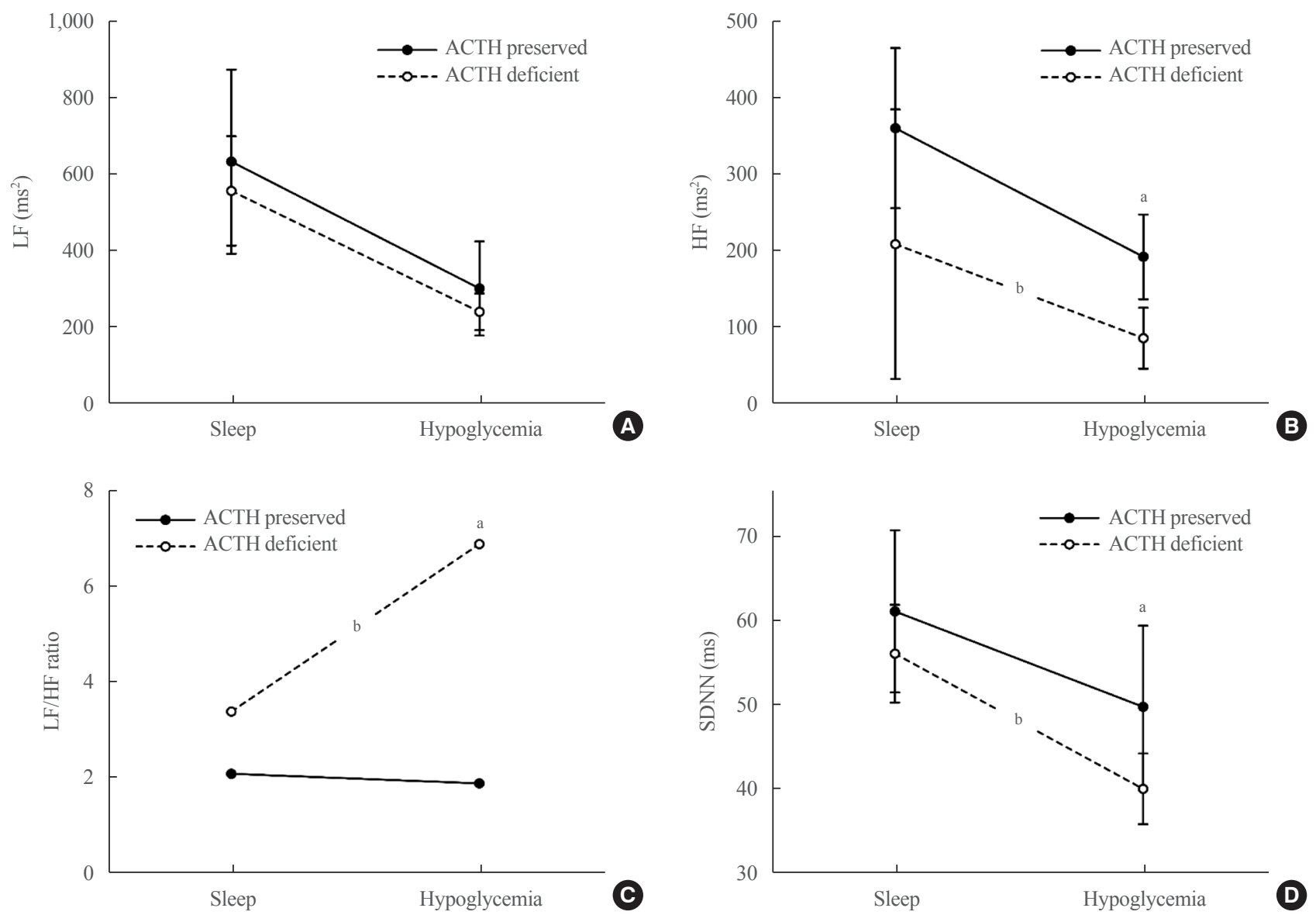

Fig. 2. Changes in heart rate variability according to cortisol status. The time of the most stable state during sleep (3:00 AM) was defined as a stable period, and the hypoglycemic period during the combined pituitary function test was defined as an unstable period. (A) Low frequency (LF). (B) High frequency (HF). (C) LF/HF ratio. (D) Standard deviation of normal-to-normal interval (SDNN). Adrenocorticotropic hormone $(\mathrm{ACTH})$ deficient indicates ACTH deficiency with other pituitary hormone deficiencies. ${ }^{\text {a }} P<0.05$ between the two groups; ${ }^{\mathrm{b}} P<0.05$ between the two phases.

tio (Fig. 2C) was higher compared to the preserved group during both the stable state (sleep) and the unstable state (hypoglycemia), and significant differences were observed during the unstable state (hypoglycemia).

\section{HRV changes during hypoglycemia and the number of associated deficiencies}

During hypoglycemia, changes in HRV parameters were analyzed according to the number of hormone deficiencies. HF and SDNN were significantly lower and the LF/HF ratio was significantly higher in patients with panhypopituitarism than in patients with multiple deficiencies (Table 4).

\section{DISCUSSION}

In this study, the effect of postoperative hypopituitarism that oc-
Table 4. Heart Rate Variability Changes According to the Number of Hormone Deficiencies during Hypoglycemia (Most Unstable State)

\begin{tabular}{lccc}
\hline Variable & $\begin{array}{c}\text { Multiple } \\
\text { deficiency }(n=10)\end{array}$ & $\begin{array}{c}\text { Panhypopituitarism } \\
(n=12)\end{array}$ & $P$ value \\
\hline Heart rate & $70.8 \pm 3.7$ & $67.0 \pm 5.0$ & 0.545 \\
LF & $354.1 \pm 106.6$ & $183.6 \pm 33.1$ & 0.164 \\
HF & $183.6 \pm 52.8$ & $84.2 \pm 40.4$ & 0.038 \\
LF/HF ratio & 3.7 & 5.8 & 0.007 \\
SDNN & $52.1 \pm 8.7$ & $36.6 \pm 2.5$ & 0.025
\end{tabular}

Values are expressed as mean \pm standard error. Hormone stimulations were confirmed by combined pituitary function test performed at median 4.4 months' post-surgery. Unit for $\mathrm{LF}$ and $\mathrm{HF}, \mathrm{ms}^{2}$ and for SDDN, ms. $P<0.05$, between the two groups.

LF, low frequency; HF, high frequency; SDNN, standard deviation of normal-to-normal interval. 
curred in postoperative NFPA patients on cardiac autonomic dysfunction was analyzed using HRV, and this study demonstrated that HRV parameters were altered in NFPA patients with hormone deficiency. In both the stable state (sleep period) and the unstable state (hypoglycemic period), inappropriate HRV responses were confirmed in patients with hormonal deficiency regardless of the type of deficiency. Furthermore, alterations in both the time domain (SDNN) and the frequency domain (HF and LF/HF ratio) were significant during hypoglycemia in patients with ACTH deficiency with other pituitary hormone deficiencies or panhypopituitarism.

HRV is noninvasive and has been extensively validated as a reliable method to provide useful information about cardiac autonomic function, serving as a robust indicator of cardiovascular risk [18]. HRV indexes including LF, HF, LF/HF ratio, and SDNN are widely analyzed for the prediction of cardiac autonomic dysfunction, and the LF/HF ratio and SDNN are recognized as representing overall autonomic function [15]. HF is an indicator reflecting parasympathetic effects [10] and increases in a stable state, particularly during sleep, but decreases in a disease state $[10,15]$. The LF/HF ratio increases with cardiac autonomic dysfunction, while SDNN decreases [15].

Hypoglycemia leads to a physiologically unstable condition, and various physiological defense mechanisms respond accordingly [19]. These defense mechanisms against hypoglycemia are impaired in patients with diabetes, and a compromised response to hypoglycemia can be correlated with impaired autonomic function [20]. The analysis of HRV changes during hypoglycemia has mainly been performed in patients with diabetes. Soydan et al. [21] found significantly reduced SDNN in patients with type 2 diabetes during hypoglycemia induced by an insulinotropic agent. Silva et al. [22] reported an increased LF/ HF ratio in moderate/severe hypoglycemic patients with type 1 diabetes. Moreover, compromised autonomic function can cause increased mortality [23-25]. However, there have been no studies analyzing HRV changes during hypoglycemia in other diseases because hypoglycemia is not naturally triggered. In the present study, a hypoglycemic state induced during the CPFT was utilized because artificially inducing hypoglycemia poses ethical issues.

The present study evaluated how HRV changes during a stable state (sleep period) and an unstable state (hypoglycemic period) in postoperative NFPA patients and how these changes depend on the type and number of hormones that are deficient. In patients with ACTH deficiency with other pituitary hormone deficiencies or panhypopituitarism, significant alterations in
HRV parameters in hypoglycemia were confirmed. Much as the $\mathrm{LF} / \mathrm{HF}$ ratio increases during hypoglycemia in patients with diabetes, the LF/HF ratio was significantly increased during hypoglycemia in patients with ACTH deficiency or panhypopituitarism. Additionally, a significant decrease in SDNN was confirmed during hypoglycemia in these patients. SDNN is lowered with autonomic dysfunction; therefore, autonomic dysfunction is anticipated in patients with ACTH deficit or panhypopituitarism. Similar results were observed in deficits of other major hormones. With GH and TSH deficiency, HF decreased, the LF/ HF ratio increased, and SDNN decreased. However, the difference was not statistically significant because of the small number of subjects.

Mortality rates in pituitary adenoma have been analyzed in hypersecretion conditions such as acromegaly or Cushing's disease; in patients with these functioning tumors, cardiometabolic risk increases due to excessive exposure to hormones [8,26,27]. However, the mortality data in NFPA are conflicting. A Swedish population-based study reported that only female NFPA patients with hypopituitarism had increased mortality rates [5], but data on the severity of pituitary dysfunction and the type of deficiency were not provided. More recently, O'Reilly et al. [7] retrospectively reviewed 519 NFPA patients and found that ACTH deficiency and Gn deficiency caused an increased risk of mortality during a 7-year follow-up. In a study in which NFPA patients accounted for $57 \%$ of subjects, Tomlinson et al. [28] reported that the excess mortality from hypopituitarism was related to the administration of supraphysiologic doses of corticosteroids and was thus attributable to cardiovascular causes. In the present study, significant HRV changes were observed during hypoglycemia in both ACTH deficiency with other pituitary hormone deficiencies and panhypopituitarism. Therefore, we hypothesized that this adverse change in HRV implies a change in cardiac autonomic balance; in particular, ACTH deficiency interferes with cardiac autonomic function and ultimately increases cardiovascular mortality.

Increased mortality in ACTH-deficient NFPA patients was reported in previous cohort studies [7,8,29]. However, the cause of the increased mortality was described as either a life-threatening adrenal crisis [30] or chronic supraphysiologic glucocorticoid replacement $[7,31,32]$. Since we stopped glucocorticoid replacement for a sufficient period before the CPFT to enable a valid interpretation of the test results, we confirmed that ACTH deficit itself poses a risk of cardiac autonomic dysfunction. In this light, the hypothesis of previous studies that adrenal crisis increases the mortality rate of NFPA patients undergoing sur- 
gery seems more convincing than the competing hypothesis. There is no research yet to confirm whether HRV parameters improve after proper glucocorticoid supplementation. Further studies are needed to confirm whether adequate glucocorticoid supplementation improves cardiac autonomic dysfunction in patients with ACTH deficiency.

Several caveats should be mentioned. First, ACTH is rarely the only deficient pituitary hormone. Rather, all states containing ACTH deficits were analyzed. Therefore, the cause of cardiac autonomic dysfunction is not the result of a single ACTH deficiency but a situation involving ACTH deficiency. Second, as acknowledged by other researchers among the limitations of HRV studies [33], there is still controversy over the analysis of the $\mathrm{LF} / \mathrm{HF}$ ratio as a means of measuring sympathovagal imbalance. However, the LF/HF ratio has been widely used as an index to indirectly evaluate sympathetic balance in previous studies, and it is currently the most available representative marker of HRV $[18,33,34]$. Third, it would be a comprehensive study to quantify patients' symptoms through a specialized questionnaire assessing quality of life in hypopituitarism and then comparing it with HRV data. Unfortunately, this content was not prepared when planning and executing the present study, and thus could not be confirmed as a parameter for clinical assessment. Finally, this study represented the cross-sectional changes in sympathetic imbalance according to changes in pituitary hormones. A long-term observational study would confirm whether adequate supplementation of the deficient hormones improves HRV.

In the present study, we confirmed by HRV analysis that significant cardiac autonomic dysfunction occurred in postoperative NFPA patients with ACTH deficiency with other pituitary hormone deficiencies or panhypopituitarism. The results of this study serve as a physiological basis for the existing analysis of increased cardiac mortality in NFPA patients and support the need for a proactive assessment of the cardiovascular risk of these patients. The present findings also suggest that adequate pituitary hormone replacement, including glucocorticoid supplementation, is essential to maintain cardiac autonomic function, although further research is needed to determine whether proper hormone supplementation improves autonomic dysfunction.

\section{CONFLICTS OF INTEREST}

The devices used in this research were provided by Taewoong Medical Co. Ltd. (Gimpo, Korea). Mr. Minsoo Yeo provided technical support in analyzing data from the HRV devices. The opinions expressed in this article do not necessarily represent those of Taewoong Medical Co. Ltd.

\section{ACKNOWLEDGMENTS}

Part of this study was accepted for an oral presentation at the 7th Seoul International Congress of Endocrinology and Metabolism, April 18 to 21, 2019 in Seoul, Korea (GO04_4). This study was supported by the Research Fund of Seoul St Mary's Hospital, The Catholic University of Korea (ZC20OISI0688). The final version has been approved by all authors.

\section{AUTHOR CONTRIBUTIONS}

Conception or design: S.H.L., J.H.C., K.H.B., D.J.L. Acquisition, analysis, or interpretation of data: J.H., H.B., C.J., M.Y. Drafting the work or revising: J.H. Final approval of the manuscript: M.I.K., D.J.L.

\section{ORCID}

Jeonghoon Ha https://orcid.org/0000-0001-9219-7135

Dong-Jun Lim https://orcid.org/0000-0003-0995-6482

\section{REFERENCES}

1. Losa M, Mortini P, Barzaghi R, Ribotto P, Terreni MR, Marzoli SB, et al. Early results of surgery in patients with nonfunctioning pituitary adenoma and analysis of the risk of tumor recurrence. J Neurosurg 2008;108:525-32.

2. Rosen T, Bengtsson BA. Premature mortality due to cardiovascular disease in hypopituitarism. Lancet 1990;336:285-8.

3. Bates AS, Van't Hoff W, Jones PJ, Clayton RN. The effect of hypopituitarism on life expectancy. J Clin Endocrinol Metab 1996;81:1169-72.

4. Erfurth EM, Bengtsson BA, Christiansen JS, Bulow B, Hagmar L. Premature mortality and hypopituitarism. Lancet 2001;357:1972.

5. Olsson DS, Nilsson AG, Bryngelsson IL, Trimpou P, Johannsson G, Andersson E. Excess mortality in women and young adults with nonfunctioning pituitary adenoma: a Swedish nationwide study. J Clin Endocrinol Metab 2015; 100:2651-8.

6. Lindholm J, Nielsen EH, Bjerre P, Christiansen JS, Hagen C, Juul S, et al. Hypopituitarism and mortality in pituitary adenoma. Clin Endocrinol (Oxf) 2006;65:51-8.

www.e-enm.org 
7. O'Reilly MW, Reulen RC, Gupta S, Thompson CA, Dineen R, Goulden EL, et al. ACTH and gonadotropin deficiencies predict mortality in patients treated for nonfunctioning pituitary adenoma: long-term follow-up of 519 patients in two large European centres. Clin Endocrinol (Oxf) 2016;85:74856.

8. Sherlock M, Reulen RC, Alonso AA, Ayuk J, Clayton RN, Sheppard MC, et al. ACTH deficiency, higher doses of hydrocortisone replacement, and radiotherapy are independent predictors of mortality in patients with acromegaly. J Clin Endocrinol Metab 2009;94:4216-23.

9. Task Force of the European Society of Cardiology and the North American Society of Pacing and Electrophysiology. Heart rate variability. Standards of measurement, physiological interpretation, and clinical use. Eur Heart J 1996;17:35481.

10. Rajendra Acharya U, Paul Joseph K, Kannathal N, Lim CM, Suri JS. Heart rate variability: a review. Med Biol Eng Comput 2006;44:1031-51.

11. Sim SY, Joo KM, Kim HB, Jang S, Kim B, Hong S, et al. Estimation of circadian body temperature rhythm based on heart rate in healthy, ambulatory subjects. IEEE J Biomed Health Inform 2017;21:407-15.

12. Kim HS, Yoon KH, Cho JH. Diurnal heart rate variability fluctuations in normal volunteers. J Diabetes Sci Technol 2014;8:431-3.

13. Park S, Kim WJ, Cho NJ, Choi CY, Heo NH, Gil HW, et al. Predicting intradialytic hypotension using heart rate variability. Sci Rep 2019;9:2574.

14. Tarvainen MP, Niskanen JP, Lipponen JA, Ranta-Aho PO, Karjalainen PA. Kubios HRV: heart rate variability analysis software. Comput Methods Programs Biomed 2014;113: 210-20.

15. Bilchick KC, Berger RD. Heart rate variability. J Cardiovasc Electrophysiol 2006;17:691-4.

16. Kim SY. Diagnosis and treatment of hypopituitarism. Endocrinol Metab (Seoul) 2015;30:443-55.

17. Melmed S. Williams textbook of endocrinology. 14th ed. Philadelphia: Elsevier Saunders; 2019. p. 184-235.

18. Billman GE. Heart rate variability: a historical perspective. Front Physiol 2011;2:86.

19. Cryer PE. Mechanisms of hypoglycemia-associated autonomic failure in diabetes. N Engl J Med 2013;369:362-72.

20. Adler GK, Bonyhay I, Failing H, Waring E, Dotson S, Freeman R. Antecedent hypoglycemia impairs autonomic cardiovascular function: implications for rigorous glycemic control. Diabetes 2009;58:360-6.

21. Soydan N, Bretzel RG, Fischer B, Wagenlehner F, Pilatz A, Linn $\mathrm{T}$. Reduced capacity of heart rate regulation in response to mild hypoglycemia induced by glibenclamide and physical exercise in type 2 diabetes. Metabolism 2013;62: 717-24.

22. Silva TP, Rolim LC, Sallum Filho C, Zimmermann LM, Malerbi F, Dib SA. Association between severity of hypoglycemia and loss of heart rate variability in patients with type 1 diabetes mellitus. Diabetes Metab Res Rev 2017;33: e2830.

23. De Ferrari GM, Sanzo A, Bertoletti A, Specchia G, Vanoli E, Schwartz PJ. Baroreflex sensitivity predicts long-term cardiovascular mortality after myocardial infarction even in patients with preserved left ventricular function. J Am Coll Cardiol 2007;50:2285-90.

24. Vinik AI, Ziegler D. Diabetic cardiovascular autonomic neuropathy. Circulation 2007;115:387-97.

25. Maser RE, Mitchell BD, Vinik AI, Freeman R. The association between cardiovascular autonomic neuropathy and mortality in individuals with diabetes: a meta-analysis. Diabetes Care 2003;26:1895-901.

26. Hassan-Smith ZK, Sherlock M, Reulen RC, Arlt W, Ayuk J, Toogood AA, et al. Outcome of Cushing's disease following transsphenoidal surgery in a single center over 20 years. J Clin Endocrinol Metab 2012;97:1194-201.

27. Nilsson B, Gustavasson-Kadaka E, Bengtsson BA, Jonsson B. Pituitary adenomas in Sweden between 1958 and 1991: incidence, survival, and mortality. J Clin Endocrinol Metab 2000;85:1420-5.

28. Tomlinson JW, Holden N, Hills RK, Wheatley K, Clayton $\mathrm{RN}$, Bates AS, et al. Association between premature mortality and hypopituitarism. West Midlands Prospective Hypopituitary Study Group. Lancet 2001;357:425-31.

29. Hannon MJ, Crowley RK, Behan LA, O'Sullivan EP, O'Brien MM, Sherlock M, et al. Acute glucocorticoid deficiency and diabetes insipidus are common after acute traumatic brain injury and predict mortality. J Clin Endocrinol Metab 2013;98:3229-37.

30. Burman P, Mattsson AF, Johannsson G, Hoybye C, Holmer H, Dahlqvist $P$, et al. Deaths among adult patients with hypopituitarism: hypocortisolism during acute stress, and de novo malignant brain tumors contribute to an increased mortality. J Clin Endocrinol Metab 2013;98:1466-75.

31. Petersons CJ, Mangelsdorf BL, Thompson CH, Burt MG. Acute effect of increasing glucocorticoid replacement dose 
on cardiovascular risk and insulin sensitivity in patients with adrenocorticotrophin deficiency. J Clin Endocrinol Metab 2014;99:2269-76.

32. Zueger T, Kirchner P, Herren C, Fischli S, Zwahlen M, Christ E, et al. Glucocorticoid replacement and mortality in patients with nonfunctioning pituitary adenoma. J Clin Endocrinol Metab 2012;97:E1938-42.

33. Comunello A, Dassie F, Martini C, De Carlo E, Mioni R,
Battocchio M, et al. Heart rate variability is reduced in acromegaly patients and improved by treatment with somatostatin analogues. Pituitary 2015;18:525-34.

34. Berntson GG, Bigger JT Jr, Eckberg DL, Grossman P, Kaufmann PG, Malik M, et al. Heart rate variability: origins, methods, and interpretive caveats. Psychophysiology 1997; 34:623-48. 